\title{
MYC-microRNA-9-metastasis connection in breast cancer
}

\author{
Maria Inês Almeida ${ }^{1,2}$, Rui M Reis ${ }^{2}$, George A Calin ${ }^{1}$ \\ ${ }^{I}$ Department of Experimental Therapeutics, The University of Texas M. D. Anderson Cancer Center, Houston, TX 77030, USA; ${ }^{2}$ Life \\ and Health Sciences Research Institute (ICVS), School of Health Sciences, University of Minho, 4710-057 Braga, Portugal \\ Cell Research (2010) 20:602-603. doi:10.1038/cr.2010.70; published online 25 May 2010
}

Metastasis accounts for more than $90 \%$ of cancer patients' mortality. The metastatic process involves multiple steps [1]. Initially, cancer cells from the primary tumor invade adjacent stroma. To acquire this capacity, cells undergo a process called epithelial-mesenchymal transition (EMT), in which cells in response to signals from the surrounding stroma, undergo a switch between cell phenotypes and acquire mesenchymal properties and show reduced intercellular adhesion, allowing cells to become motile. Then cells enter systemic circulation, either through the blood or lymph, and finally extravasate into the parenchyma of distant tissues, where they form micrometastasis and proliferate to form secondary tumors [2].

MicroRNAs (miRNAs) are a class of non-coding RNAs with approximately $22 \mathrm{nt}$ length that regulate gene expression post-transcriptionally by binding to 3' untranslated region (UTR), coding sequence or $5^{\prime}$ UTR of target messenger RNAs (mRNAs), leading to inhibition of translation or mRNA degradation [3]. Tumors present aberrant expression of miRNAs patterns. miRNAs have been described as regulators of several biological processes such as apoptosis, proliferation, differentiation, and more recently, metastasis [4]. miRNAs can function either as suppressors or pro-

\footnotetext{
Correspondence: George A Calin

Tel: 713-792-5461; Fax: 713-745-4528

E-mail: gcalin@mdanderson.org
}

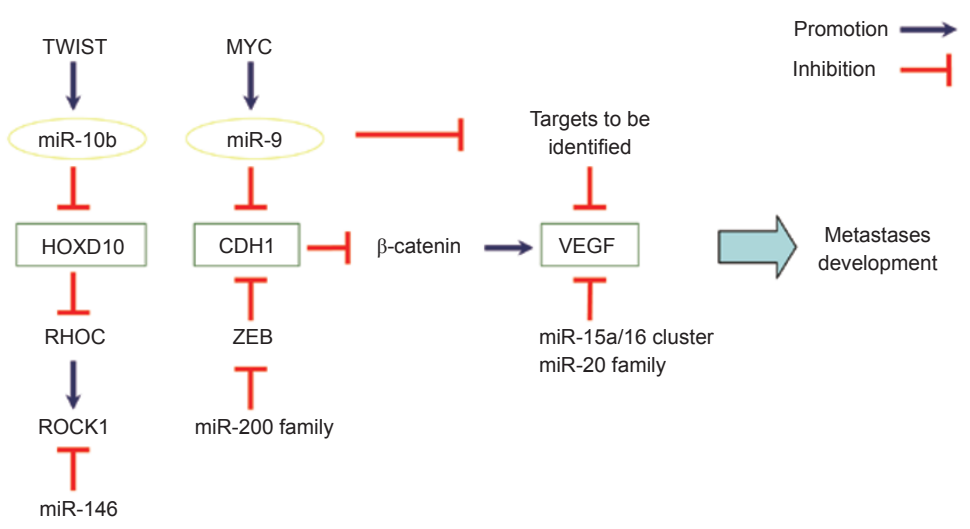

Figure 1 Two small non-coding RNAs (miR-9 and miR-10b) and the complex regulatory network of cancer metastasis (data from ref $[2,5,6]$ ).

moters of metastasis according to their mRNA targets $[1,2]$. Elucidation of the role of miRNAs in metastasis can lead to a better understanding of this process and consequently lead to new treatments for patients with late-stage tumors.

In 2007, Ma and coworkers from Weinberg's group reported the first study about the role of miRNAs in tumor metastasis [5]. The authors identified three miRNAs, namely miR-155, miR-9 and miR-10b, as being upregulated in breast cancer cell lines and showed that miRNA-10b, by targeting HOXD10a, induce the pro-metastatic gene $R H O C$ and consequently contribute to metastasis [5]. In a recent publication in Nat Cell Biol [6], Ma and colleagues elegantly show the involvement of miR-9 in the metastasis-regulating signaling network (Figure 1). A common approach to identify miRNA targets is the use of computational algorithms whereby the authors identify and further confirm by luciferase assay and western blot that the cell-cell adhesion receptor E-cadherin (encoded by $\mathrm{CDH} 1$ ) is a direct target of miR-9. This miRNA binds to $\mathrm{CDH} 13^{\prime} \mathrm{UTR}$ region as a decrease in luciferase activity was detected in cells co-transfected with miR-9 and CDH1 3'UTR reporter gene. To further confirm this result, the authors show that cotransfection of miR-9 and CDH1 3'UTR reporter gene containing a mutation in the seed-target region do not cause a decrease in luciferase activity [6].

This finding is important because, as it has long been recognized, loss of E-cadherin expression is one of the hallmarks of EMT process [7]. E-cadherin is a transmembrane protein whose primary function is to mediate cell adhesion. The cytoplasmic tail of E-cadherin as- 
sociates with several proteins including $\beta$-catenin. Besides its involvement in cell adhesion, $\beta$-catenin is an essential effector of the Wnt signaling pathway which is frequently upregulated in tumors [8]. Specifically, $\beta$-catenin forms a transcription factor complex with TCF to regulate gene expression. When $\beta$-catenin escapes degradation, it accumulates in the cytosol and can enter the nucleus where it will act as a transcriptional co-regulator $[7,8]$. miR-9 upregulation in breast cancer cells suppresses E-cadherin that consequently loses its capacity to sequester $\beta$-catenin and potentiates the Wnt signaling. In fact, the authors show that miR-9 expression increases $\beta$-catenin activity in cell lines with a basal E-cadherin expression. In vivo, miR-9 overexpressing tumor xenografts develop more micrometastasis than tumor xenografts without miR-9 overexpression. In addition, in vivo, inhibition of miR-9 can decrease metastasis formation [6]. Silencing of miR-9 and modulating E-cadherin expression may represent a new therapeutic approach in advanced breast cancers to prevent metastasis formation.

Study by Ma et al. is exciting because the authors demonstrate the contribution of miR-9 not only for EMT but also for induction of angiogenesis that is essential for metastasis to develop. VEGFA is a key pro-angiogenic protein and a target of $\beta$-catenin [9]. Ma et al. show that miR-9 overexpression upregulates VEGFA and that it correlates with E-cadherin expression. In vivo, the same result is obtained: VEGFA plasma levels in miR-9-overexpressing tumor xenografts are higher than in controls indicating the role of miR-9 in tumor angiogenesis. However, by itself Ecadherin and $\beta$-catenin do not induce VEGFA upregulation [6]. Therefore, other miR-9 targets have to be considered. In future studies it will be interesting to answer the question what other proteins/pathways may mediate VEGFA upregulation via miR-9 overexpression. Finally, Ma et al. explore upstream pathway of miR-9 expression regulation and find out its activation by MYC/ MYCN. The authors correlate miR-9 upregulation with MYC amplification in neuroblastomas tumor samples and also with metastatic breast tumors [6]. However, the number of breast cancer samples used in the study is reduced and so it remains to be determined if miR-9 can be used as a prognostic marker since large breast cancer association studies are needed for this. In conclusion, this study convincingly identifies a new player in both EMT and angiogenic processes - miR-9, and opens intriguing new avenues of diagnostic and therapy: Do miR-9 levels in tumors and/or plasma of breast cancer patients predict metastasis? Should anti-miR-9 (and anti-miR-10b) be used for metastases prophylaxis in cancer patients?

Currently the role of miRNAs as one of key regulators of the metastatic process is starting to be clarified. Similar to miRNAs, other non-coding RNAs (ncRNAs) were shown to be deregulated in tumors [10]. In the future, it will be interesting to address the role of other ncRNAs in the complex molecular puzzle of metastasis.

\section{References}

1 Hurst DR, Edmonds MD, Welch DR. Metastamir: the field of metastasisregulatory microRNA is spreading. Cancer Res 2009; 69:7495-7498.

2 Nicoloso MS, Spizzo R, Shimizu M, Rossi S, Calin GA. MicroRNAs--the micro steering wheel of tumour metastases. Nat Rev Cancer 2009; 9:293-302.

3 Bartel DP. MicroRNAs: genomics, biogenesis, mechanism, and function. Cell 2004; 116:281-297.

4 Spizzo R, Nicoloso MS, Croce CM, Calin GA. SnapShot: MicroRNAs in Cancer. Cell 2009; 137:586-586.e1.

5 Ma L, Teruya-Feldstein J, Weinberg RA. Tumour invasion and metastasis initiated by microRNA-10b in breast cancer. Nature 2007; 449:682-688.

6 Ma L, Young J, Prabhala H, et al. miR-9, a MYC/MYCN-activated microRNA, regulates E-cadherin and cancer metastasis. Nat Cell Biol 2010; 12:247-256.

7 Jeanes A, Gottardi CJ, Yap AS. Cadherins and cancer: how does cadherin dysfunction promote tumor progression? Oncogene 2008; 27:6920-6929.

8 Fodde R, Brabletz T. Wnt/beta-catenin signaling in cancer stemness and malignant behavior. Curr Opin Cell Biol 2007; 19:150-158.

9 Skurk C, Maatz H, Rocnik E, Bialik A, Force T, Walsh K. Glycogen-Synthase Kinase3beta/beta-catenin axis promotes angiogenesis through activation of vascular endothelial growth factor signaling in endothelial cells. Circ Res 2005; 96:308-318

10 Calin GA, Liu CG, Ferracin M, et al. Ultraconserved regions encoding ncRNAs are altered in human leukemias and carcinomas. Cancer Cell 2007; 12:215229. 